\title{
RESEARCH
}

Open Access

\section{The addition of neoadjuvant pertuzumab for the treatment of HER2+ breast cancer: a cost estimate with real-world data}

\author{
Andreia Borges ${ }^{1,2}$, Filipa Pereira ${ }^{3}$, Patrícia Redondo ${ }^{1,2}$, Luís Antunes ${ }^{4,5}$, Cláudia Vieira ${ }^{3,6,7}$, Pedro Antunes ${ }^{8}$, \\ Maria José Bento ${ }^{4,5,9}$, Susana Sousa ${ }^{3}$, José Machado Lopes ${ }^{1}$, Francisco Rocha-Gonçalves ${ }^{10}$, \\ Joaquim Abreu de Sousa ${ }^{8}$, Deolinda Sousa Pereira ${ }^{3}$ and Marina Borges ${ }^{1,2^{*}}$ (D)
}

\begin{abstract}
Background: Breast cancer (BC) is largely prevalent worldwide. HER2-positive BC account for roughly 20-25\% of all BC cases and has an overall survival lower than other BC. Innovation on BC therapeutics is a constant, but novel therapies have higher costs. Therefore, cost-effectiveness research is essential to provide healthcare decision-makers with solid foundations for a resource allocation. This study aims to estimate the average direct medical costs/ patient and cost-effectiveness of adding pertuzumab in neoadjuvant treatment (NeoT) for HER2-positive breast cancer (BC).

Methods: Two retrospective real-world consecutive cohorts of $\geq 18 y$ o female patients diagnosed with HER2positive BC treated with NeoT at the Breast Clinic of IPO-Porto were studied. The AC-DH regimen (2012-2015) comprised 8 cycles of neoadjuvant therapy ( 4 cycles of doxorubicin + cyclosphosphamide followed by 4 cycles ofdocetaxel + trastuzumab), while the AC-DHP regimen (2015-2017) included also pertuzumab as NeoT. NeoT was followed by surgery and adjuvant trastuzumab. Micro-costing technique and a bottom-up approach was used comprising all medical direct costs from the hospital perspective. Unit costs were obtained from government official prices or from IPO-Porto costing system. Costs were adjusted to 2017 and are expressed in euros. Multivariable logistic regression models were used for effectiveness assessment, while generalized linear models with gamma distribution were used for costs. ICER was calculated using the pathological complete response (pCR) as the preferential measure of effectiveness. Sensitivity analysis was also performed.

Results: AC-DHP $(n=40)$ and AC-DH $(n=54)$ cohorts had heterogenous patient profiles (median age 43y/53y; $67.5 \% / 59.3 \%$ positive HR; $60.0 \% / 27.8 \%$ operable; $25.0 \% / 24.1 \%$ inflammatory, respectively). The AC-DHP average total cost/patient was 56,375€, with pertuzumab accounting for $13,978 €(24.79 \%)$ and increasing in $15,982 €$ the average cost/patient $(p<0.001)$. Clinical staging and hormone receptors $(H R)$ were significantly associated with $\mathrm{pCR}$. ICER was $1.370 €$ per percentage point of $\mathrm{pCR}$.
\end{abstract}

\footnotetext{
* Correspondence: marina.borges@ipoporto.min-saude.pt

'Outcomes Research Lab, Portuguese Oncology Institute of Porto (IPO Porto), Porto, Portugal

${ }^{2}$ Management, Outcomes Research, and Economics in Healthcare Group, IPO Porto Research Center (CI-IPOP), Portuguese Oncology Institute of Porto (IPO Porto), Porto, Portugal

Full list of author information is available at the end of the article
}

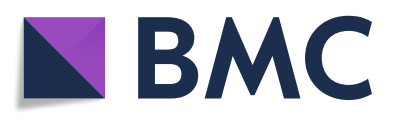

(c) The Author(s). 2021 Open Access This article is licensed under a Creative Commons Attribution 4.0 International License, which permits use, sharing, adaptation, distribution and reproduction in any medium or format, as long as you give appropriate credit to the original author(s) and the source, provide a link to the Creative Commons licence, and indicate if changes were made. The images or other third party material in this article are included in the article's Creative Commons licence, unless indicated otherwise in a credit line to the material. If material is not included in the article's Creative Commons licence and your intended use is not permitted by statutory regulation or exceeds the permitted use, you will need to obtain permission directly from the copyright holder. To view a copy of this licence, visit http://creativecommons.org/licenses/by/4.0/. The Creative Commons Public Domain Dedication waiver (http://creativecommons.org/publicdomain/zero/1.0/) applies to the data made available in this article, unless otherwise stated in a credit line to the data. 
Conclusions: ICER was more favourable in stage III HR negative BC patients compared to other patient profiles. Innovative treatments access is critical to deliver high-quality healthcare, but sustainability must be considered. These results suggest the importance of establishing a cost-effectiveness profile of Pertuzumab in NeoT for HER2positive BC.

\section{Highlights}

i. The results obtained in the AC-DHP cohort, which included Pertuzumab as a NeoT, suggest better clinical outcomes compared to the AC-DH group, a confirmation based on real-world data.

ii. Some patient profiles seem to be more promising concerning the overall costs for pathological complete response, but the present data does not include a long-term follow-up period, which suggests that interpretations should be performed with caution.

iii. Pertuzumab cost estimated represented a great proportion of overall total costs per patient, but further research (including the costs of the whole episode of care) and continuously monitoring (additional RWE) is needed for a more robust cost-effectiveness analysis.

Keywords: Pertuzumab, Neoadjuvant treatment, HER2-positive breast cancer, Cost-effectiveness analysis

\section{Background}

Breast cancer $(\mathrm{BC})$ is the most common cancer in women in the world. It affects more than 2 million women globally each year and represents approximately $15 \%$ of female cancer deaths [1]. In Portugal, $\mathrm{BC}$ was responsible, in 2018 , for $12 \%$ of all cancer cases and was the leading cause of cancer in women, reaching an incidence of $128.7 /$ 100,000 [2]. Death rate data from 2017 puts Portugal (34.4 deaths per 100,000) behind its neighbour Spain (28.5 deaths per 100,000), but ahead of other wealthy nations like France, Germany and the United Kingdom [3]. However, the disease was still responsible for 3.4\% (3.2-3.6\%) of all female deaths in Portugal in 2017 [3].

Like in other cancers, the histological subtypes in $\mathrm{BC}$ impact in the therapeutic approach and in the expected clinical outcomes. The overexpression of the human epidermal growth factor receptor (HER) 2 protein, commonly known as HER2-positive $\mathrm{BC}$, is present in approximately $20-25 \%$ of $\mathrm{BC}$ cases and is associated with worse prognosis and, consequently, overall survival (OS) [4].

The treatment success in HER2-positive BC was improved with the use of trastuzumab as neoadjuvant and adjuvant therapy. In fact, the introduction of trastuzumab has transformed the natural history of the disease for these patients [5]. Nonetheless, still approximately $25-30 \%$ of HER2-positiveBC treated patients still experience relapse during follow-up after adjuvant therapy with trastuzumab $[5,6]$. In this sense, new therapeutic options, such as pertuzumab, have been developed [7].
Pertuzumab is an anti-HER2 monoclonal antibody initially approved for the treatment of patients with HER2positive metastatic or locally recurrent unresectable $\mathrm{BC}$. Later, in 2015, the indication was extended and comprised the neoadjuvant treatment (NeoT) of adult patients with HER2-positive BC, locally advanced, inflammatory, or early stage $\mathrm{BC}$ at high risk of recurrence [8]. A second extension of the indication was approved in 2018 for the adjuvant treatment of early $\mathrm{BC}$ at high risk of recurrence and metastatic $\mathrm{BC}$ [9].

Neoadjuvant pertuzumab has shown positive efficacy and safety results [10,11], and promising progressionfree survival and disease-free survival after 5 years [12]. However, in terms of health economic data, available literature is mainly based on economic models populated with clinical trial data and estimations of resource utilization [13-18], demonstrating the necessity of generating real-world data (RWD) on pertuzumab utilization. In Portugal, the local authority INFARMED has not yet published a Health Technology Assessment (HTA) appraisal of pertuzumab for the NeoT of HER2positive BC in early stages. Nonetheless, patients might have access to treatment under special authorization and case by case review by INFARMED.

Considering the scenario of increasing health expenses and resources constraints in National Health System (NHS), a reference public large-sized comprehensive cancer center plays an important role in monitoring cancer treatments effectiveness and in estimating its costs, so assessing the impact of a new technology is necessary 
not only for hospital budgets, but for HTA bodies. The assessment of the drug performance according to an institution's clinical practice is, therefore, important for assessing the real benefit of a newly implemented technology, so that new payment models based on its outcomes can be implemented, in a value-based healthcare (VBHC) perspective. Hence, patients can benefit from new technology while assuring the sustainability of the NHS, which is currently a major concern.

To our best knowledge, the cost per patient of pertuzumab in neoadjuvant context was not yet calculated using real-world patient level data, thus this research aims to estimate the average direct medical costs per patient by adding pertuzumab in the clinical practice to the NeoT of HER2-positive BC patients, as well as to calculate the weight of pertuzumab in the overall costs. Additionally, this study also aims to explore costeffectiveness results of real-world patients treated with pertuzumab in neoadjuvant context.

\section{Methods}

This study included two independent retrospective realworld consecutive cohorts of female patients aged $\geq 18$ years old diagnosed with HER-2 positive BC that received the NeoT between 2012 and 2017 at the Portuguese Oncology Institute of Porto (IPO-Porto) and followed at the institution's Breast Clinic. IPO-Porto is a national reference public hospital located in the North of Portugal and specialized in the treatment of cancer. The hospital has 11 multidisciplinary specialty units (Porter's Integrated Practice Unit - IPU), including the Breast Clinic. This clinic started its activities in 2007 and has an independent functional structure with its own areas and specialized staff, allowing patients to receive an integrated and personalized care. In 2018, received 1690 new cases, for a total of approximately 11,000 patients in follow-up, corresponding roughly to $10 \%$ of all IPO-Porto patients. Overall, the IPO-Porto BC unit is responsible for treating the greatest number of $\mathrm{BC}$ patients in Portugal and is one of the largest $\mathrm{BC}$ units of Europe.

Patients were consecutively included in the study with the diagnosis of operable, locally advanced, or inflammatory stage II or III HER2-positive unilateral BC who performed the complete NeoT at IPO-Porto between January 2012 and mid-2017 were included in the current analysis. All patients received 4 cycles of doxorubicin plus cyclophosphamide followed by 4 cycles of docetaxel. In 2012-2015 period, the regimen included 4 cycles of trastuzumab (AD-DH cohort), while in 2015-2017 (ACDHP cohort) the patients received 4 cycles of dual HER2-blockade with pertuzumab and trastuzumab as a NeoT in accordance with approved Summary of Product Characteristics [7]. For all patients, the NeoT was followed by surgery and trastuzumab as adjuvant therapy every 21 days, as described in Fig. 1 . All patients being treated at the center with one of these protocols between 2012 and 2017 matching inclusion criteria were included in the study.

Exclusion criteria includes patients with stages I or IV at baseline or that performed other chemotherapy treatment rather than above mentioned treatment protocol or that had other cancer diagnosis during study period besides unilateral $\mathrm{BC}$ were excluded. Males, clinical trials participants, patients that underwent partial treatment in another institution and non-naive patients for $\mathrm{BC}$ chemotherapy were also excluded. Also, patients with other oncological pathology, and that did not perform the complete pathway at IPO Porto were also excluded. Matching between cohorts was not performed.

This research received approval from the Local Ethics Committee, followed all National ethical standards and procedures, and was performed in accordance with the Helsinki declaration.

\section{Data collection}

Demographic, clinical, treatment and costs data were collected using Excel. The confidentiality and anonymization of data for analysis were ensured.

Data on demographics (age, weight and height), clinical staging/TMN classified by AJCC 7th edition [19], hormone receptors (HR) (positive if estrogen and/or progesterone receptor was $>1 \%$; HER2 was positive if the score was $3+$ in immunohistochemistry or in case of $2+$ positive by fluorescence in situ hybridization (FISH), Eastern Cooperative Oncology Group (ECOG) Performance Status [20], systemic treatment (date, type of treatment, drug, dose), surgical treatment (mastectomy or conservative, axillary clearance, biopsy of sentinel lymph node, number of positive lymph nodes), and pathological complete response (pCR) as measure of treatment effectiveness were collected. Tumours were classified as operable (T0-3, N-0-1, M0), locally advanced (T2-3, N2-3, M0 or T4a-c, any N, M0) or inflammatory (T4d, any N, M0) following the same criteria from the NeoSphere trial [10].

Cost data information regarding chemotherapy, hormone therapy (includes drugs consumed from outpatient pharmacy and sessions of hospital day), radiotherapy, hospital appointments and exams (includes scheduled consultations, emergency appointments and complementary diagnostic exams), inpatient and outpatient care (includes surgical outpatient care and IPO-Porto homestay costs) were collected to estimate the direct medical costs.

The date of diagnosis was considered as the baseline date and as the starting point for the data collection and medical resource consumption accountability; and the 


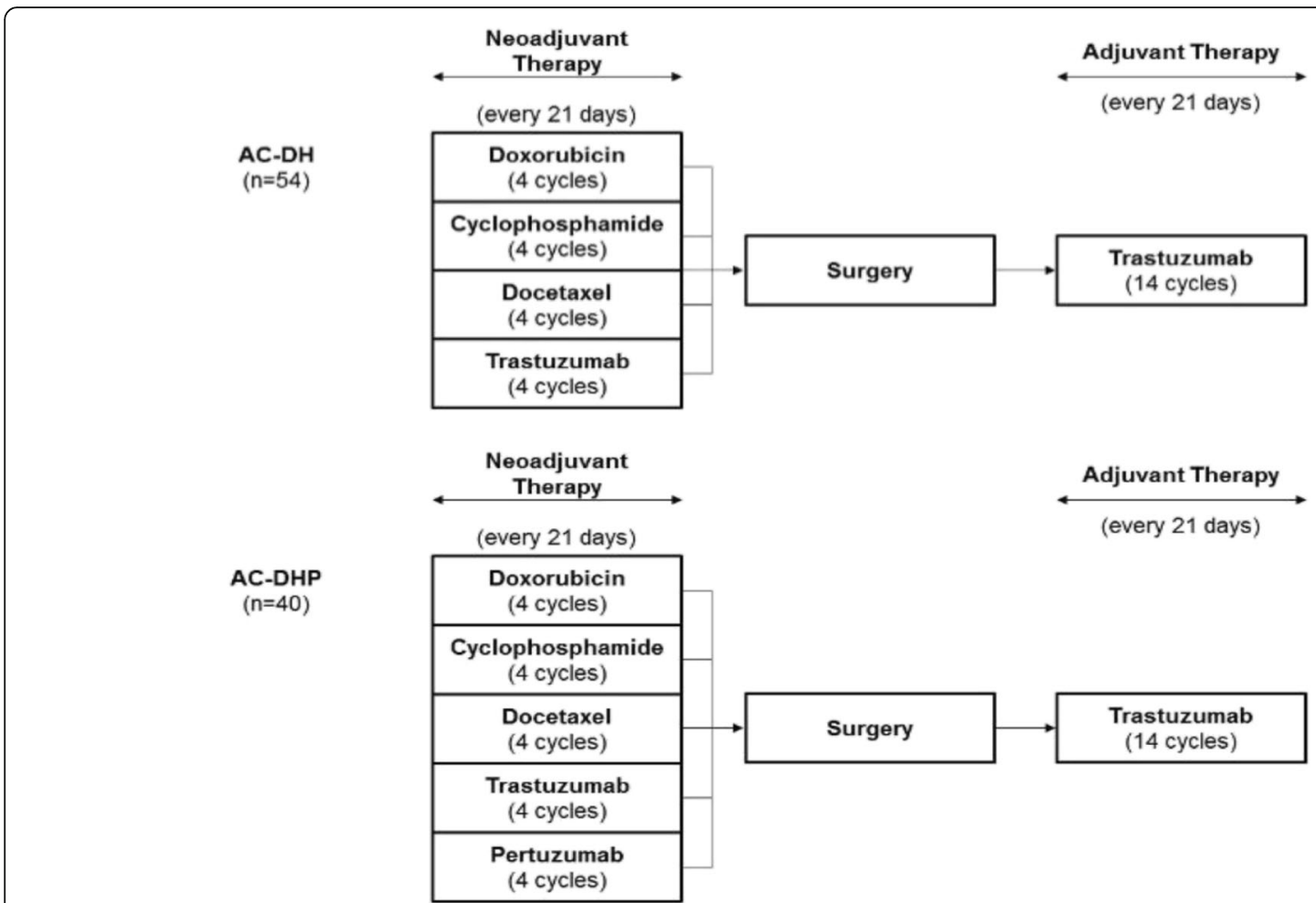

Fig. 1 Schematic representation of the pertuzumab treatment protocol. AC-DH: 2012-2015 cohort; A: doxorubicin; C: cyclophosphamide; DH: docetaxel plus trastuzumab; AC-DHP - 2015-2017 cohort; A: doxorubicin; C: cyclophosphamide; DHP - docetaxel, trastuzumab plus pertuzumab

last adjuvant administration of trastuzumab was considered the end of data collection.

\section{Cost estimation}

The micro-costing technique was used, and all medical direct costs from the date of diagnosis to the date of the last administration of adjuvant treatment were accounted individually for each patient.

The unit costs were obtained from government official prices [21-23], except for the drug prices that were obtained from the IPO-Porto costing system - which follows the approved prices by the drug regulator for public hospital drugs (Infarmed). The cost per milligram was calculated and multiplied by the quantity of milligrams administrated per session. All costs were considered for the year of 2017, but hospitalizations/inpatient care and outpatient care episodes were adjusted for this same year using the following formula:

Current price $=$ Price in legislation $\times(1+$ Variation from current price $)$

By using unit costs, the authors assumed that the unit cost does not differ by patient. For example, the unit cost of a consultation is the same regardless of the duration, even though it may take longer in the beginning of the adjuvant therapy or for older patients. This source of variability was not take into account in the present study.

The outpatient care episodes also include the IPOPorto homestay costs estimated based on the government official prices.

To determine the total cost per patient, the costs accrued for each variable were summed. The average, median, minimum and maximum cost per patient was obtained for both cohorts. The total cost was calculated by the sum of the costs for each treated patient.

Only medical direct costs from the hospital perspective were considered. Costs were expressed in euros for the year of 2017. The discount rate is not applicable.

\section{Health outcomes}

The clinical outcome measured was the rate of $\mathrm{pCR}$ on breast and axilla achieved in last assessment after surgery. The rate was measured by the percentage of patients that achieved the pCR. The pCR is defined as the absence of invasive neoplastic cells or in-situ lesions (ypT0/is ypN0) and is associated with an improved survival, being a suitable outcome for measuring NeoT effectiveness in HER2 positive BC treatment [24, 25].

\section{Study outcomes}

For the main analysis, the average, median, maximum and minimum total cost per patient was calculated for 
both cohorts. The proportion of the pertuzumab cost in the total costs was calculated by dividing the mean pertuzumab cost by the mean total costs per patient, the mean total chemotherapy costs and the mean of total neoadjuvant costs.

The Incremental Cost-Effectiveness Ratio (ICER) was also estimated by the following formula:

$$
\mathrm{ICER}=\frac{\text { Average total costs }_{\mathrm{AC}-\mathrm{DHP}}-\text { Average total costs }_{\mathrm{AC}-\mathrm{DH}}}{\mathrm{pCR} \text { Rate }_{\mathrm{AC}-\mathrm{DHP}}-\mathrm{pCR} \text { Rate }_{\mathrm{AC}-\mathrm{DH}}}
$$

\section{Statistical analysis}

Descriptive statistics for categorical variables included frequencies' tabulation with counts and percentages, and for continuous variables comprised central tendency and dispersion measures. Comparisons between the two cohorts were performed using independent samples t-test for continuous variables. Chi-square or Fisher's exact tests were used to evaluate the association between two categorical variables.

Binary logistic regression models were used to quantify the relation between patient's characteristics and pCR. Age, stage of disease at diagnosis, hormone receptors, ECOG, Body Mass Index (BMI), and type of surgery were analysed. Regimen was forced into the optimized model to ensure proper comparison. Exponentiated coefficients (adjusted Odds Ratios (OR)), $p$-value, and 95\% confidence intervals $(\mathrm{CI})$ were calculated.

Generalized linear model (GLM) with gamma distribution was used for modelling continuous skewed treatment costs per patient, allowing to test the mean cost increase. All independent variables were included in the model as categorical. The optimized model was obtained by stepwise backward elimination method for selection of independent variables. The adjusted mean cost ratio (MCR), $p$-value, and 95\% CI were calculated.

For the binary logistic regression models and for the generalized linear models, first, univariate models were performed and each variable was considered separately. Then, the variables that were considered statistically significant in the univariate models were retained for the multivariable model. The final multivariable model contains the variables that were statistically significant.

A $p$-value $<0.05$ was considered statistically significant. Data were computed using STATA V.15 for Windows (StataCorp).

\section{Sensitivity analysis}

The coefficients estimated from multivariable logistic regression were used to perform sensitivity analysis. Sensitivity analysis was performed varying the clinical staging and the HR, obtaining, for each scenario tested, the ICER, the cost per pCR, the number of patients who needed to be treated to have a $\mathrm{PCR}$ and the additional cost for $\mathrm{pCR}$. Four scenarios were tested: clinical staging II and negative HR, clinical staging II and positive HR, clinical staging III and negative HR, and clinical staging III and positive HR. The average total direct cost per patient obtained from main analysis was used in all four tested scenarios.

\section{Results}

\section{Baseline characterization}

A total of 40 patients (average age 45.4 years, $67.5 \%$ with HR positive, $60.0 \%$ with operable and $25.0 \%$ with inflammatory tumour) that performed the AC-DHP regimen were included in the analysis. For the AC-DH cohort, a total of 54 patients (average age 50.9 years, $59.3 \%$ with HR positive, $27.8 \%$ with operable and $24.1 \%$ with inflammatory tumour) were included. Baseline characteristics are described in Table 1.

\section{Health outcomes}

The clinical outcomes are described in Table 2. A total of $45.0 \%$ of patients treated with AC-DHP regimen presented pCR in breast and axilla, while only $33.3 \%$ of patients treated with AC-DH regimen had the same response. Although approximately $35 \%$ higher, the $\mathrm{pCR}$ difference between both cohorts was not statistically significant for the sample size analysed $(p>0.05)$.

pCR separately by breast and axilla were additionally calculated and the results for the pertuzumab cohort were also clinically better in both breast and axilla (pCR in breast: $50 \%$ vs. $40.7 \%$; pCR in axilla: $60 \%$ vs. $48.1 \%$ ).

Optimized logistic regression model is presented in Table 3. The treatment regimen was not significantly associated with the $\mathrm{pCR}$ in the breast and axilla (ypT0/is ypN0) and $\mathrm{pCR}$ in the breast $(p=0.304$ and $p=0.396$, respectively), however the association was positive. On the other hand, clinical staging and positive HR were the only patient's characteristics significantly associated with the achievement of $\mathrm{pCR}$ in the breast and axilla $(p=$ 0.001 and $p=0.003$, respectively) and $\mathrm{pCR}$ in the breast ( $\mathrm{p}=0.001$ and $p<0.001$, respectively). The authors opted to present only the variables that were identified as statistically significant predictors and the treatment regimen, which although not significant, was the main variable of interest in this study.

\section{Study outcomes - costs comparison between cohorts}

Mean, median, maximum and minimum total cost per patient for both treatment cohorts are presented in Table 4 and additional graphical representation is presented in Supplementary Fig. 1. Cost comparison was performed between groups and Total cost of session of hospital day, Drug cost for complete regimen and Drug cost for neoadjuvant regimen, associated with Chemotherapy/ Targeted therapy, as well as Conservative treatment cost (Inpatient 
Table 1 Patients demographics and clinical characteristics at baseline

\begin{tabular}{|c|c|c|c|}
\hline & $\begin{array}{l}\text { Doxorubicin, cyclophosphamide, docetaxel, } \\
\text { trastuzumab plus pertuzumab }(n=40)\end{array}$ & $\begin{array}{l}\text { Doxorubicin, cyclophosphamide, } \\
\text { docetaxel plus trastuzumab }(n=54)\end{array}$ & $P$-value \\
\hline Age in years (average \pm standard deviation) & $45.4 \pm 9.6$ & $50.9 \pm 9.9$ & 0.008 \\
\hline \multicolumn{4}{|l|}{ Age group (n, \%) } \\
\hline$\leq 24$ & $1(2.5 \%)$ & $0(0.0 \%)$ & \multirow[t]{10}{*}{0.068} \\
\hline $25-29$ & $0(0.0 \%)$ & $1(1.9 \%)$ & \\
\hline $30-34$ & $2(5.0 \%)$ & $1(1.9 \%)$ & \\
\hline $35-39$ & $8(20.0 \%)$ & $7(13.0 \%)$ & \\
\hline $40-44$ & $12(30.0 \%)$ & $4(7.4 \%)$ & \\
\hline $45-49$ & $5(12.5 \%)$ & $12(22.2 \%)$ & \\
\hline $50-54$ & $5(12.5 \%)$ & $7(13.0 \%)$ & \\
\hline $55-59$ & $3(7.5 \%)$ & $12(22.2 \%)$ & \\
\hline $60-64$ & $2(5.0 \%)$ & $4(7.4 \%)$ & \\
\hline$\geq 65$ & $2(5.0 \%)$ & $6(11.1 \%)$ & \\
\hline BMI (average \pm standard deviation) & $25.5 \pm 4.7$ & $27.0 \pm 5.7$ & 0.168 \\
\hline \multicolumn{4}{|l|}{ ECOG performance status [20] (n, \%) } \\
\hline 0 & $34(85.0 \%)$ & $47(87.0 \%)$ & \multirow[t]{2}{*}{0.777} \\
\hline 1 & $6(15.0 \%)$ & $7(13.0 \%)$ & \\
\hline \multicolumn{4}{|l|}{ Hormonal Receptors (ER and PR) (n, \%) } \\
\hline At least one positive & $27(67.5 \%)$ & $32(59.3 \%)$ & \multirow[t]{2}{*}{0.414} \\
\hline Both negative & $13(32.5 \%)$ & $22(40.7 \%)$ & \\
\hline \multicolumn{4}{|l|}{ Clinical staging ( $n, \%)$} \\
\hline$\| \mathrm{A}$ & $4(10.0 \%)$ & $2(3.7 \%)$ & \multirow[t]{5}{*}{0.205} \\
\hline$\| \mathrm{B}$ & $8(20.0 \%)$ & $8(14.8 \%)$ & \\
\hline IIIA & $18(45.0 \%)$ & $22(40.7 \%)$ & \\
\hline IIIB & $10(25.0 \%)$ & $17(31.5 \%)$ & \\
\hline IIIC & $0(0.0 \%)$ & $5(9.3 \%)$ & \\
\hline \multicolumn{4}{|l|}{ Histologic grade (n, \%) } \\
\hline G1 & $0(0.0 \%)$ & $1(1.9 \%)$ & \multirow[t]{4}{*}{0.640} \\
\hline G2 & $11(27.5 \%)$ & $19(35.2 \%)$ & \\
\hline G3 & $28(70.0 \%)$ & $32(59.2 \%)$ & \\
\hline Undetermined & $1(2.5 \%)$ & $2(3.7 \%)$ & \\
\hline \multicolumn{4}{|l|}{ At diagnosis $(n, \%)$} \\
\hline Operable & $24(60.0 \%)$ & $15(27.8 \%)$ & \multirow[t]{3}{*}{0.001} \\
\hline Locally advanced & $6(15.0 \%)$ & $26(48.1 \%)$ & \\
\hline Inflammatory & $10(25.0 \%)$ & $13(24.1 \%)$ & \\
\hline \multicolumn{4}{|l|}{ Lymph node status ( $\mathrm{n}, \%)$} \\
\hline No & $4(10.0 \%)$ & $10(18.5 \%)$ & \multirow[t]{4}{*}{0.006} \\
\hline N1 & $27(67.5 \%)$ & $18(33.3 \%)$ & \\
\hline N2 & $9(22.5 \%)$ & $21(38.9 \%)$ & \\
\hline N3 & $0(0.0 \%)$ & $5(9.3 \%)$ & \\
\hline \multicolumn{4}{|l|}{ Tumour size (n, \%) } \\
\hline T0 & $1(2.5 \%)$ & $0(0.0 \%)$ & \multirow[t]{5}{*}{0.420} \\
\hline $\mathrm{T} 1$ & $2(5.0 \%)$ & $2(3.7 \%)$ & \\
\hline $\mathrm{T} 2$ & $13(32.5 \%)$ & $11(20.4 \%)$ & \\
\hline T3 & $14(35.0 \%)$ & $21(38.9 \%)$ & \\
\hline T4 & $10(25.0 \%)$ & $20(37.0 \%)$ & \\
\hline
\end{tabular}


Table 2 Clinical outcomes for HER2-positive breast cancer by regimen

\begin{tabular}{llll}
\hline & $\begin{array}{l}\text { Doxorubicin, cyclophosphamide, docetaxel, } \\
\text { trastuzumab plus pertuzumab }(\boldsymbol{n = 4 0 )}\end{array}$ & $\begin{array}{l}\text { Doxorubicin, cyclophosphamide, } \\
\text { docetaxel plus trastuzumab }(\boldsymbol{n}=\mathbf{5 4})\end{array}$ & $\begin{array}{l}\boldsymbol{P} \text {-value } \\
\text { (chi square test) }\end{array}$ \\
\hline Pathological complete response & & \\
ypT0/is ypNO & $18(45.0 \%)$ & $18(33.3 \%)$ & 0.250 \\
Breast & $20(50.0 \%)$ & $22(40.7 \%)$ & 0.372 \\
Axilla & $24(60.0 \%)$ & $26(48.1 \%)$ & 0.255 \\
\hline
\end{tabular}

Data are number (\%). DCIS, ductal carcinoma in situ. $\mathrm{nCR}$, near complete response

care), were found to be significantly higher in the ACDHP cohort. Conversely, Goserelin cost (Hormone Therapy), Total radiotherapy costs and Simple technique cost (Radiotherapy) were higher for the AC-DH cohort. As expected, overall costs were significantly higher in the ACDHP cohort.

Pertuzumab cost alone was on average $13,978 €$ per patient, which represents $24.8 \%$ of the overall costs per patient $(56,375 €), 34.8 \%$ of the average cost per patient for complete drug regimen $(40,217 €)$ and $67.5 \%$ of the overall neoadjuvant therapy costs $(20,695 €)$. The average cost of all chemotherapy drugs represented the greatest portion of the total costs, reaching $71.3 \%$ of the complete treatment costs. The hormone therapy, radiotherapy, appointments, exams, inpatient care, including surgery, and outpatient care were responsible for the $28.7 \%$ of the remaining costs. The scenario before pertuzumab followed this trend, with chemotherapy total cost $(25,818 €)$ representing the greatest portion of all treatment costs $(40,393 €)$, reaching $63.9 \%$.
The addition of pertuzumab increased in $15,982 €$ the mean cost per patient, of which $2004 €$ represented all other medical resources consumed excluding pertuzumab. The adoption of AC-DHP regimen at IPO-Porto increased in $39.6 \%$ the overall costs per patient, with $4.7 \%$ of the cost increased explained by the other medical resources.

\section{Sensitivity analysis}

From the GLM models we obtained a statistically significant mean cost increase of $40 \%$. Patient's treatment costs were explained by regimen and, for this reason, sensitivity analysis was conducted only varying the clinical staging and HR. The ICER, as well as sensitivity analysis performed are shown in Table 5. By calculating the ICER, we can conclude that, to increase the $\mathrm{pCR}$ rate by one percentage (pp) point, we need to spend on average $1370 €$.

Regarding the sensitivity analysis and comparing all the scenarios, lower clinical staging had better results.

Table 3 Optimized Predictive Analysis for pathological complete response and treatment cost per patient

\begin{tabular}{|c|c|c|c|c|}
\hline \multicolumn{5}{|c|}{ Predictors of pathological complete response (Multivariable logistic regression) } \\
\hline & \multicolumn{2}{|c|}{ Pathological complete response ypTO/is ypNO } & \multicolumn{2}{|c|}{ Pathological complete response in the breast } \\
\hline & Adjusted OR (95\% Cl) & $P$-value & Adjusted OR (95\% Cl) & P-value \\
\hline \multicolumn{5}{|l|}{ Regimen } \\
\hline $\mathrm{AC}-\mathrm{DH}$ & 1 & & 1 & \\
\hline AC-DHP & $1.65(0.63-4.30)$ & 0.304 & $1.51(0.58-3.94)$ & 0.396 \\
\hline \multicolumn{5}{|l|}{ Clinical staging } \\
\hline$\|$ & 1 & & 1 & \\
\hline III & $0.14(0.04-0.43)$ & 0.001 & $0.14(0.04-0.44)$ & 0.001 \\
\hline \multicolumn{5}{|c|}{ Hormonal Receptors (ER and PR) } \\
\hline Both negative & 1 & & 1 & \\
\hline At least one positive & $0.21(0.08-0.59)$ & 0.003 & $0.16(0.06-0.43)$ & $<0.001$ \\
\hline
\end{tabular}

Predictors of treatment cost per patient (Gamma General Model)

\section{Cost per patient}

Regimen

Adjusted MCR $(95 \% \mathrm{Cl})$

AC-DH

AC-DHP

$1.40(1.30-1.49)$
P-value

AC-DH, doxorubicin, cyclophosphamide, docetaxel plus trastuzumab. AC-DHP, doxorubicin, cyclophosphamide, docetaxel, trastuzumab plus pertuzumab. ER, estrogen receptor. $\mathrm{PR}$, progesterone receptor 


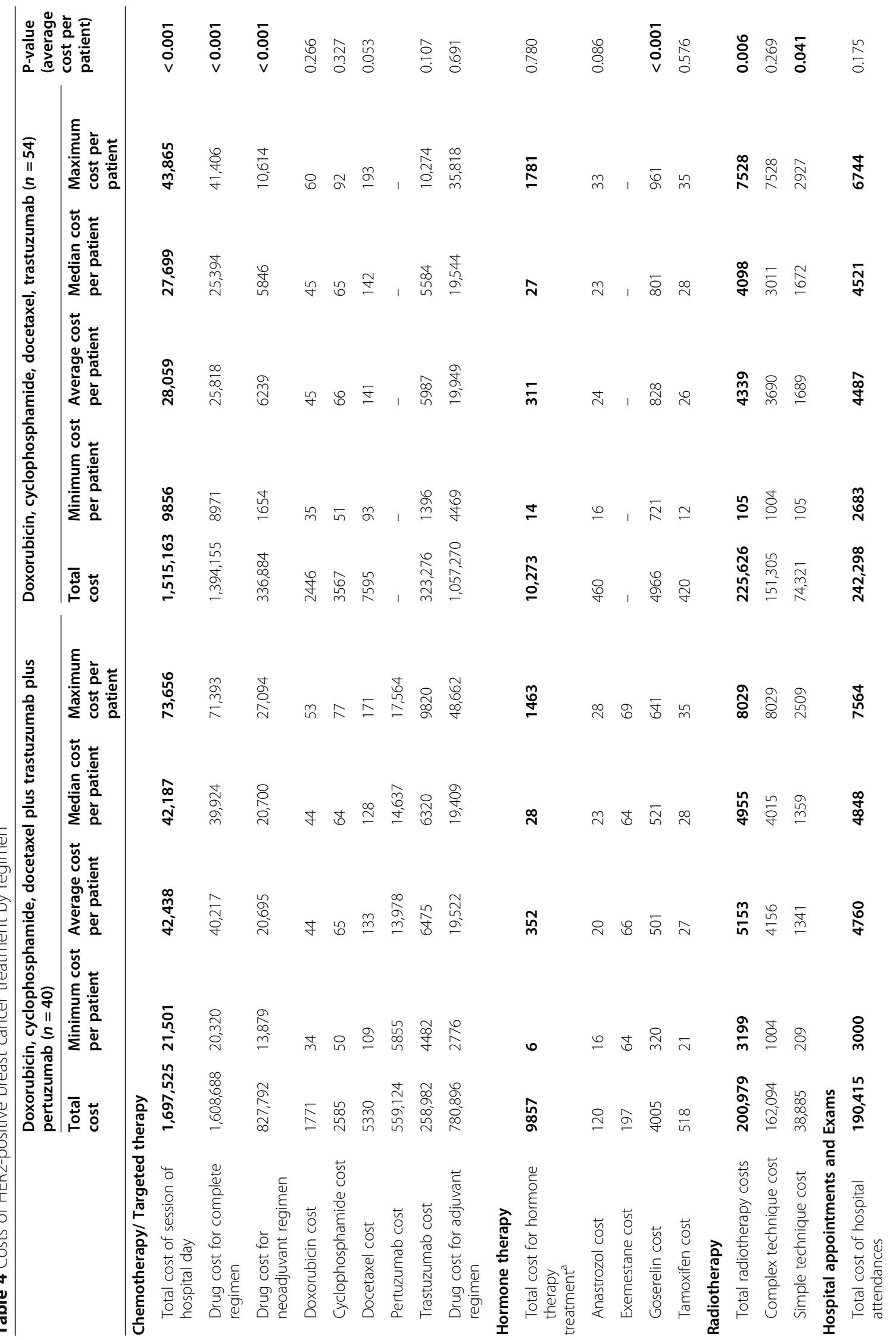




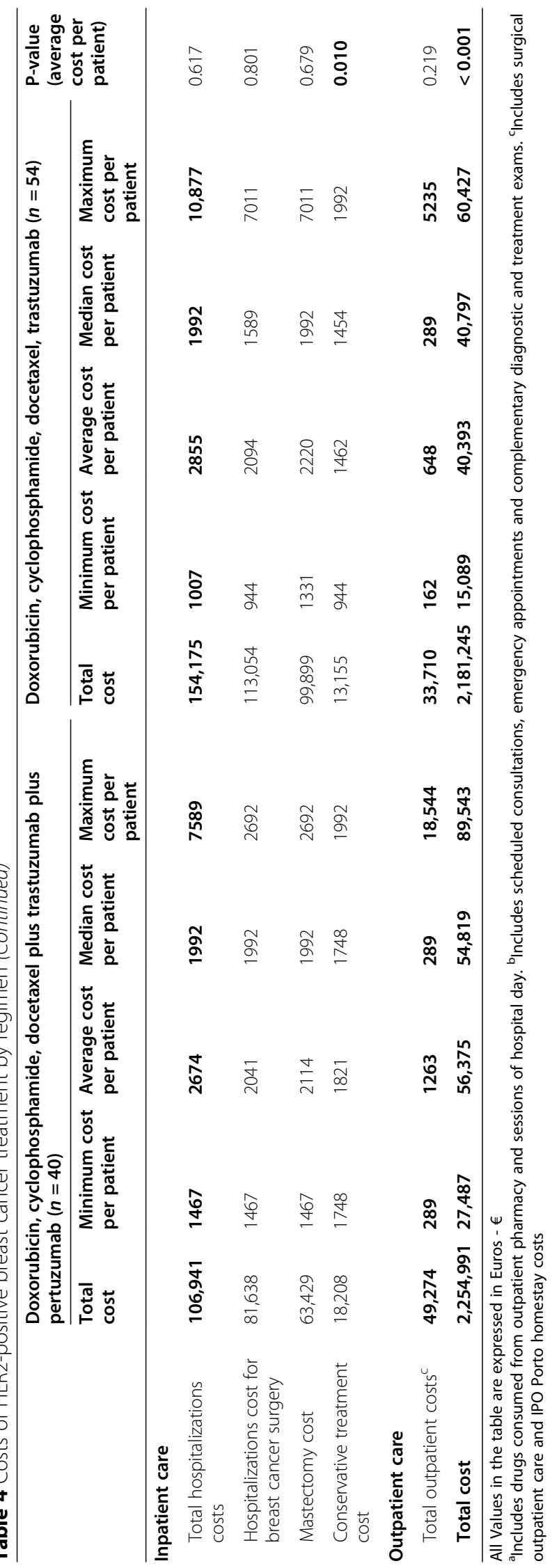


Table $\mathbf{5}$ Incremental cost-effectiveness ratio (ICER) by regimen and sensitivity analysis

\begin{tabular}{|c|c|c|c|c|c|c|}
\hline Regimen & Effectiveness & $\begin{array}{l}\text { Total direct } \\
\text { cost per } \\
\text { patient }\end{array}$ & ICER & $\begin{array}{l}\text { Cost per } \\
\text { pathological } \\
\text { complete response }\end{array}$ & $\begin{array}{l}\text { No. of patients who needed to } \\
\text { be treated to have a pathological } \\
\text { complete response }\end{array}$ & $\begin{array}{l}\text { Additional cost } \\
\text { for pathological } \\
\text { complete response } \\
\end{array}$ \\
\hline AC-DH & $33 \%$ & $40,393 €$ & & $121,180 €$ & 3.0 & \\
\hline AC-DHP & $45 \%$ & $56,375 €$ & $1370 €$ & $125,277 €$ & 2.2 & $4097 €$ \\
\hline \multicolumn{7}{|c|}{ Sensitivity analysis } \\
\hline \multicolumn{7}{|c|}{ Scenario 1. Clinical staging II and negative HR } \\
\hline AC-DH & $85 \%$ & $40,393 €$ & & $47,640 €$ & 1.2 & \\
\hline AC-DHP & $90 \%$ & $56,375 €$ & $2955 €$ & $62,502 €$ & 1.1 & $14,862 €$ \\
\hline \multicolumn{7}{|c|}{ Scenario 2. Clinical staging II and positive HR } \\
\hline AC-DH & $54 \%$ & $40,393 €$ & & $74,493 €$ & 1.8 & \\
\hline AC-DHP & $66 \%$ & $56,375 €$ & $1338 €$ & $85,205 €$ & 1.5 & $10,711 €$ \\
\hline \multicolumn{7}{|c|}{ Scenario 3. Clinical staging III and negative HR } \\
\hline AC-DH & $43 \%$ & $40,393 €$ & & $93,682 €$ & 2.3 & \\
\hline AC-DHP & $56 \%$ & $56,375 €$ & $1282 €$ & $101,427 €$ & 1.8 & $7745 €$ \\
\hline \multicolumn{7}{|c|}{ Scenario 4. Clinical staging III and positive HR } \\
\hline AC-DH & $14 \%$ & $40,393 €$ & & $291,147 €$ & 7.2 & \\
\hline AC-DHP & $21 \%$ & $56,375 €$ & $2241 €$ & $268,371 €$ & 4.8 & $-22,775 €$ \\
\hline
\end{tabular}

AC-DH, doxorubicin, cyclophosphamide, docetaxel plus trastuzumab. AC-DHP, doxorubicin, cyclophosphamide, docetaxel, trastuzumab plus pertuzumab. HR, hormonal receptors (include progesterone and estrogen). ICER, incremental cost-effectiveness ratio

Moreover, the presence of negative HR is considerably associated with better treatment results when compared to positive HR. Clinical staging II and negative HR had the best effectiveness results for both treatment protocols, but the highest ICER. In this case, to increase the pCR rate by one percentage point, it is necessary to spend on average $2955 €$. For the same clinical staging and positive HR, the treatment effectiveness for both cohorts was lower compared to negative HR however the ICER was lower $(1338 €)$.

Regarding clinical staging III, the trend was the same: the presence of positive HR was associated with less treatment effectiveness. However, a more advanced clinical staging inverted the ICER: $1282 €$ for negative HR and $2241 €$ for positive HR.

\section{Discussion}

Technological advances in the diagnosis and treatment of $\mathrm{BC}$ have not solved the risk of recurrence and high mortality rate of HER2-positive BC. New available treatment options, such as pertuzumab, must be continuously assessed for safety and effectiveness after marketing authorization, which also includes costeffectiveness. Increasing healthcare costs and resource constraints in the Portuguese NHS [26], lead to debate highly expensive cancer drugs [27]. Assessing the real benefit and price of new drugs is extremely important to the NHS and society as a whole to ensure better resource allocation. Thus, cost-effectiveness and continuous drug performance assessments are important for improving sustainable access to high-value care for patients in need.

Pertuzumab as a NeoT for HER2-positive BC is a relative novelty. Since it is a new treatment, the scarcity of real-world evidence on cost and effectiveness is noticeable. Present results show that patients treated with pertuzumab (AC-DHP protocol) had better clinical outcomes (but non-significant), measured as pCR rates in breast and axilla and pCR rates in breast only, which is in line with the clinical trials results (clinical value) $[10,12]$. On the other hand, our study was, to the best of our knowledge, the first to describe a detailed bottom-up analysis of the $\mathrm{BC}$ treatment in a public healthcare institution and explore cost-effectiveness of pertuzumab in the real-world setting (economic value).

Accordingly, pertuzumab as NeoT for HER2-positive $\mathrm{BC}$ is costly and averages almost one fourth of all treatment costs, one third of chemotherapy and targeted therapy drug costs and more than two thirds of the NeoT costs. Our results are in line with previous research [28] who estimated the real-world treatment cost of metastatic HER2-positive BC from 2004 to 2010 and found that trastuzumab and chemotherapy represented the most significant part of all treatment costs. Pertuzumab was not available in that time frame, but immunotherapy drugs cost weighted heavily in the overall costs. Interestingly, our study identified that the average total cost of chemotherapy and targeted therapy alone in the 
AC-DHP cohort $(42,438 €)$ was higher than the complete treatment cost in the AC-DH cohort $(40,393 €)$.

The bottom-up approach in this study accurately and comprehensively estimated the institution's costs with the treatment of HER2-positive BC. Unlike direct costs estimation based on expert opinion or resources consumed in clinical trials, our study relies on a robust and realistic quantification of current treatment costs per patient.

Only direct medical costs are reported here (indirect medical costs were not estimated), which may have led to an underestimation of the overall treatment cost. Data on patient's follow-up over a longer time horizon was unavailable, since patients in the last cohort (AC-DHP) received NeoT with pertuzumab in 2018. Assessing the impact of $\mathrm{BC}$ relapse on costs and survival using RWD was, therefore, not possible.

Nevertheless, the estimated costs are a valuable information for IPO-Porto budgeting and for future HTA assessments in defining drug prices based on its value. In fact, these estimated costs could be used to populate cost-effectiveness models with RWD and accurately estimate simulated long-term horizons with transitions between health states. Our study could ultimately be complemented by indirect costs estimations, additional costs associated with $\mathrm{BC}$ relapse and health-related quality of life (HRQOL) data in patients with $\mathrm{BC}$-obtained from current literature- providing better costeffectiveness and cost-utility treatment assessment.

Concerning the mean healthcare expenditures per patient/year, the estimated costs are impressive versus average healthcare costs in Portugal. The average estimated overall cost per patient of the AC-DHP cohort $(56,375 €)$ was 35 times higher than the Portuguese national mean healthcare expenditure per patient/year (1630.05€ in 2016) [29]. Nevertheless, the abovementioned cost underestimation -more evident in a societal perspective- is enough to highlight the high economic burden of $\mathrm{BC}$ treatments. This should raise awareness on the importance of technology assessments and resources allocation concerning NHS sustainability.

As for clinical outcomes, pCR results were not statistically significant. This can probably be explained by sample size constraints and baseline differences between cohorts. In addition, the long term benefits of using pertuzumab to reduce $\mathrm{BC}$ relapse and increase progressionfree survival were not evaluated in this research, despite the important insights on costs.

The sensitivity analysis considered variations in the effectiveness of different sub-groups, changing the clinical staging and positive/negative ER and PR in each tested scenario. Patients with negative HR had better overall clinical results compared with patients with positive HR, which is in line with available literature [30]. The clinical staging II with negative HR resulted in an higher ICER versus clinical staging II with positive $H R$. This might suggest that adding pertuzumab for the treatment of clinical staging II with negative HR may not bring significant cost-effectiveness benefits when compared with positive HR cases in the same clinical staging. However, we must consider that long-term scenarios can generate better results for pertuzumab considering the reduction of receding and increasing PFS.

Conversely, the results in clinical staging III patients showed a higher ICER for positive HR cases, although the effectiveness was considerably different in favour of patients with HR negative in the clinical staging III. Pertuzumab may not be as advantageous for the treatment of clinical staging III patients with positive HR, as in the case of negative HR patients, but broader samples are needed. Broader samples size would also allow for results with greater statistical impact. Nonetheless, this limitation could not be avoided since the sample considered represented all eligible patients from IPO-Porto in the selected period. Since this is based on RWE data, stratification and baseline adjustment of characteristics were limited. As treatment protocols evolve over time and become more complex, based on available evidence, patient profile changes. Baseline differences between both cohorts limited some clinical comparisons. Protocol evolution can also explain a $5.7 \%$ cost increase when not accounting for pertuzumab, despite costs adjustment to the same year.

Additionally, although IPO-Porto is a reference center for cancer treatment, present results depict the clinical practice of a single institution, which may not be a full representation of the Portuguese context. Using specific IPO-Porto reference prices can be seen as a limitation, but since used for both treatment cohorts, it has no impact in the results.

Despite these limitations, higher clinical effectiveness of AC-DHP cohort in all scenarios and optimized model results with controlled baseline characteristics indicate clinically meaningful but non-significant results in favour of pertuzumab. However, this also stresses the need for more RWE for pertuzumab as NeoT. These results are a stepping stone in the RWE monitoring of cost-effectiveness for pertuzumab as a NeoT. Future research in collaboration with other national and international institutions should aim for more robust results by increasing sample size and the observation time. In the context of breast cancer, larger time horizons are best suited for exploring different outcomes, such as overall survival and impacts beyond healthcare, in the economic and social context of patients. IPO-Porto mission is aligned with current VBHC trends and patient centricity is at the core of the therapeutic approach. Future research should also collect Patient Reported 
Outcome Measures (such as HRQOL), which are vital to better decision-making in healthcare resource allocation.

Public oncology hospitals, like IPO-Porto, play a relevant societal role by providing specialized care for a devastating disease and by encouraging policy making and payment based on patient outcomes. This is an ongoing process done in collaboration with different stakeholders for better decision making, so patients can ultimately benefit from innovative but cost-effective therapies.

Neoadjuvant pertuzumab indication was approved in Europe in 2015 and, from an economic perspective, it received different recommendations from reference HTA bodies. The National Institute for Health and Care Excellence (NICE) in the UK [31] positively recommended the use of pertuzumab for NeoT, but received a negative recommendation from The National Centre for Pharmacoeconomics (NCPE) in Ireland [32]. Portuguese INFARMED is yet to publish the HTA appraisal for pertuzumab in NeoT.

\section{Conclusion}

Our study is important for the monitoring of pertuzumab treatment costs and its performance in the realworld context, considering the growing concern with the NHS sustainability. Although this new technology represents a significant investment, and safety and effectiveness should be continuously monitored, the availability of this treatment is very positive for clinical outcomes. Incorporating innovative therapies is essential for the quality of the universal health coverage in Portugal. Additionally, our study gave insights of the cost-effectiveness of adding neoadjuvant pertuzumab in clinical practice. Future research using pertuzumab as comparator for newly treatments in the HTA and reimbursement assessment based on patient's outcomes in Portugal will surely consider the present results.

\begin{abstract}
Abbreviations
ACT: Adjuvant chemotherapy; BC: Breast cancer; Cl: Confidence intervals; ECOG: Eastern Cooperative Oncology Group; ER: Estrogen receptor; FISH: Fluorescence in situ hybridization; GLM: Generalized linear model; HER2: Human epidermal growth factor receptor 2; HR: Hormonal receptors; HRQOL: Health-related quality of life; HTA: Health Technology Assessment; ICER: Incremental Cost-Effectiveness Ratio; nCR: Near complete response; NCPE: The National Centre for Pharmacoeconomics; NeoT: Neoadjuvant treatment; NHS: National Health System; NICE: The National Institute for Health and Care Excellence; OR: Adjusted Odds Ratios; OS: Overall survival; pCR: Pathological complete response; PFS: Progression-free survival; RWD: Real world data; VBHC: Value-Based Healthcare
\end{abstract}

\section{Supplementary Information}

The online version contains supplementary material available at https://doi. org/10.1186/s13561-021-00332-0.

Additional file 1: Supplemental Fig. 1. Treatment cost with/without pertuzumab and average cost per patient. AC-DH, adriamycin, cyclophosphamide, docetaxel plus trastuzumab. AC-DHP, adriamycin, cyclophosphamide, docetaxel, trastuzumab plus pertuzumab.

\section{Acknowledgements}

The authors would like to acknowledge Diogo Morais and Luiza Freitas from CTI Clinical Trial \& Consulting Services for the support in writing this paper.

\section{Authors' contributions}

All authors made significant contribution in the study design, data acquisition and analysis, and all agreed the final version of the manuscript. Moreover, all authors thoroughly and critically revised the manuscript before submission.

\section{Funding}

This article is a result of the project NORTE-01-0145-FEDER-000027, supported by Norte Portugal Regional Operational Program (NORTE 2020), under the PORTUGAL 2020 Partnership Agreement, through the European Regional Development Fund (ERDF). The study was independently performed by IPO-

Porto. CTI Clinical Trial \& Consulting Services was appointed by IPO-Porto for medical writing and editorial support.

\section{Availability of data and materials}

The datasets used and/or analysed during the current study are available from the corresponding author on reasonable request.

\section{Declarations}

Ethics approval and consent to participate

This research received approval from the Local Ethics Committee (Comissão de Ética para a Saúde do Instituto Português de Oncologia do Porto FG IPO-PORTO), followed all National ethical standards and procedures, and was performed in accordance with the Helsinki declaration.

\section{Consent for publication}

Not applicable.

\section{Competing interests}

FP received financial support from Roche, Merck and Pfizer. PR, SS, JAS and DSP received financial support from Roche. CV received financial support from Roche, MSD, Novartis, Pfizer, Astrazeneca, Merck Serono, Grünenthal S.A. and Laboratorios Vitória. MB received financial support from Roche and Janssen. No financial support received was related to this article. AB, LA, PA, $M J B, J M L$, FRG report no conflict of interests.

\section{Author details}

'Outcomes Research Lab, Portuguese Oncology Institute of Porto (IPO Porto), Porto, Portugal. ${ }^{2}$ Management, Outcomes Research, and Economics in Healthcare Group, IPO Porto Research Center (CI-IPOP), Portuguese Oncology Institute of Porto (IPO Porto), Porto, Portugal. ${ }^{3}$ Medical Oncology Department, Portuguese Oncology Institute of Porto (IPO Porto), Porto, Portugal. ${ }^{4}$ Department of Epidemiology, Portuguese Oncology Institute of Porto (IPO Porto), Porto, Portugal. ${ }^{5}$ Cancer Epidemiology Group, IPO Porto Research Center (CI-IPOP), Portuguese Oncology Institute of Porto (IPO Porto), Porto, Portugal. ${ }^{6}$ Molecular Oncology Group, IPO Porto Research Center (CI-IPOP), Portuguese Oncology Institute of Porto (IPO Porto), Porto, Portugal. ${ }^{7}$ Faculty of Medicine, University of Porto, Porto, Portugal. ${ }^{8}$ Surgical Oncology Department, Portuguese Oncology Institute of Porto (IPO Porto), Porto, Portugal. ${ }^{9}$ Department of Population Studies, Institute of Biomedical Sciences Abel Salazar, University of Porto, Porto, Portugal. ${ }^{10}$ Luz Saúde, Porto, Portugal.

Received: 21 January 2021 Accepted: 27 August 2021

Published online: 10 September 2021

References

1. World Health Organization. WHO | Breast cancer. World Heal. Organ. World Health Organization; 2018 [cited 2019 Apr 4]. Available from: https://www. who.int/cancer/prevention/diagnosis-screening/breast-cancer/en/

2. International Agency for Research on cancer. Portugal Globocan 2018. 2018. Available from: http://gco.iarc.fr/today/data/factsheets/populations/620portugal-fact-sheets.pdf

3. The Institute for Health Metrics and Evaluation. GBD Compare | IHME Viz Hub. 2016 [cited 2019 Apr 4]. Available from: https://vizhub.healthdata.org/ gbd-compare/ 
4. Cronin KA, Harlan LC, Dodd KW, Abrams JS, Ballard-Barbash R. Populationbased estimate of the prevalence of HER-2 positive breast cancer tumors for early stage patients in the US. Cancer Investig. 2010;28(9):963-8. https://doi. org/10.3109/07357907.2010.496759.

5. Moasser MM, Krop IE. The evolving landscape of HER2 targeting in breast cancer. JAMA Oncol. American Medical Association; 2015 [cited 2019 May 10];1:1154-61. Available from: http://oncology.jamanetwork.com/article.a spx?doi=10.1001/jamaoncol.2015.2286, 1, 8, 1154, 1161

6. Mota JM, Collier KA, Costa RLB, Taxter T, Kalyan A, Leite CA, et al. A comprehensive review of heregulins, HER3, and HER4 as potential therapeutic targets in cancer. Oncotarget. Impact J. 2017;8:89284-306 Available from: http://www.oncotarget.com/fulltext/18467.

7. CHMP. Perjeta - Annex I Summary Of Product Characteristics. 2019. Available from: https://www.ema.europa.eu/en/documents/product-information/ perjeta-epar-product-information_en.pdf.

8. Committee for Medicinal Products for Human Use (CHMP). Perjeta Summary of opinion (post authorisation) - EMA/CHMP/334570/2015. 2015. Available from: www.ema.europa.eu/contact

9. Committee for Medicinal Products for Human Use (CHMP). Perjeta Summary of opinion (post authorisation) - EMA/258941/2018. 2018. Available from: www.ema.europa.eu/contact

10. Gianni L, Pienkowski T, Im Y-H, Roman L, Tseng L-M, Liu M-C, et al. Efficacy and safety of neoadjuvant pertuzumab and trastuzumab in women with locally advanced, inflammatory, or early HER2-positive breast cancer (NeoSphere): a randomised multicentre, open-label, phase 2 trial. Lancet Oncol. 2012;13:25-32. [cited 2019 may 10] Available from: http://www.ncbi. nlm.nih.gov/pubmed/22153890.

11. Schneeweiss A, Chia S, Hickish T, Harvey V, Eniu A, Hegg R, Tausch C, Seo $J$ H, Tsai YF, Ratnayake J, McNally V, Ross G, Cortés J Pertuzumab plus trastuzumab in combination with standard neoadjuvant anthracyclinecontaining and anthracycline-free chemotherapy regimens in patients with HER2-positive early breast cancer: a randomized phase II cardiac safety study (TRYPHAENA). Ann Oncol; 2013;24:2278-2284. Available from: https://a cademic.oup.com/annonc/article-lookup/doi/10.1093/annonc/mdt182, 9

12. Gianni L, Pienkowski T, Im Y-H, Tseng L-M, Liu M-C, Lluch A, et al. 5-year analysis of neoadjuvant pertuzumab and trastuzumab in patients with locally advanced, inflammatory, or early-stage HER2-positive breast cancer (NeoSphere): a multicentre, open-label, phase 2 randomised trial. Lancet Oncol.; 2016;17:791-800. [cited 2019 Apr 1] Available from: http://www.ncbi. nlm.nih.gov/pubmed/22153890.

13. Attard CL, Pepper AN, Brown ST, Thompson MF, Thuresson P-O, Yunger S, et al. Cost-effectiveness analysis of neoadjuvant pertuzumab and trastuzumab therapy for locally advanced, inflammatory, or early HER2positive breast cancer in Canada. J Med Econ. 2015;18:173-88. [cited 2019 may 10] Available from: http://www.ncbi.nlm.nih.gov/pubmed/25347449.

14. Garrison LP, Babigumira J, Tournier C, Goertz HP, Lubinga SJ, Perez EA. Costeffectiveness analysis of Pertuzumab with Trastuzumab and chemotherapy compared to Trastuzumab and chemotherapy in the adjuvant treatment of HER2-positive breast Cancer in the United States. Value Heal. 2019:7-14.

15. Babigumira JB, Santos E, Antao VP, Wang B, Portera CC, Kamath T, et al. Projecting the cost-effectiveness of pertuzumab with trastuzumab and docetaxel in the neoadjuvant treatment of HER2-positive, locally advanced, inflammatory or early breast cancer. J Clin Oncol. Am Soc Clin Oncol; 2014; 32:642-642. [cited 2019 May 10] Available from: http://ascopubs.org/doi/1 0.1200/jco.2014.32.15_suppl.642

16. Squires H, Pandor A, Thokala P, Stevens JW, Kaltenthaler E, Clowes M, et al. Pertuzumab for the neoadjuvant treatment of early-stage HER2-positive breast Cancer: an evidence review group perspective of a NICE single technology appraisal. Pharmacoeconomics. 2018;36(1):29-38. Available from: http://eprints.whiterose.ac.uk/119850/. https://doi.org/10.1007/s40273-0170556-7.

17. Ignatyeva, Khachatryan G. Cost-Utility Analysis of Neoadjuvant Pertuzumab and Trastuzumab in Patients with Locally Advanced, Inflammatory, or Early HER-2 Positive Breast Cancer. Value Heal.; 2016;19:A739--A740. Available from: https://linkinghub.elsevier.com/retrieve/pii/S1098301516336130

18. Ribeiro, Lourenço O, Alves C, Thuresson P, Monteiroa, Batel-Marques F. Economic Evaluation of Pertuzumab In Combination with Trastuzumab and Docetaxel in the Neoadjuvant Treatment of Women with HER2-Positive, Locally Advanced, Inflammatory or Early Breast Cancer in Portugal. Value Heal; 2016;19:A736--A737. Available from: https://linkinghub.elsevier.com/ retrieve/pii/S1098301516335975
19. American Cancer Society. AJCC CANCER STAGING MANUAL - 7th edition [Internet]. AJCC Cancer Staging Handb. New York, NY: Springer New York; 2010. Available from: http://cancerstaging.org/references-tools/ deskreferences/Documents/AJCC\%207th\%20Ed\%20Cancer\%20Staging\%2 OManual.pdf.

20. ECOG-ACRIN Cancer Research Group. ECOG Performance Status. [cited 2019 Sep 3]. Available from: https://ecog-acrin.org/resources/ecogperformance-status

21. Diário da República. Portaria no 207/2017. Diário da República, 1.a série N.o 132 - 11 de julho de 2017. 2017; Available from: https://data.dre.pt/eli/ port/207/2017/07/11/p/dre/pt/html.

22. Diário da República. Portaria no 839-A/2009. Diário da República n. 147/ 2009, 10 Suplemento, Série I de 2009-07-31. 2009; Available from: https://da ta.dre.pt/eli/port/839-a/2009/07/31/p/dre/pt/html.

23. Diário da República. Portaria no 20/2014. Diário da República n.` 20/2014, Série I de 2014-01-29. 2014; Available from: https://data.dre.pt/eli/port/20/2 014/01/29/p/dre/pt/html.

24. Pennisi A, Kieber-Emmons T, Makhoul I, Hutchins L. Relevance of pathological complete response after neoadjuvant therapy for breast cancer. Breast Cancer Basic Clin Res. 2016;10:103-6 Available from: https://www.ncbi.nlm.nih.gov/pmc/articles/PMC4961053/pdf/bcbcr-10-201 6-103.pdf.

25. Hoffmann F. Application for the addition of Perjeta (pertuzumab) on the WHO model list of essential medicines. 2018. Available from: https://www. who.int/selection_medicines/committees/expert/22/applications/s8.2_ pertuzumab.pdf?ua=1

26. DRE - Diário da República Eletrónico. Diário da República n.o 60/2018, 10 Suplemento, Série II de 2018-03-26. 2018. Available from: https://dre.pt/ home/-/dre/114925355/details/maximized?serie=II\&dreld=114925353.

27. Cancer Research UK. Health economics: the cancer drugs cost conundrum; 2016.

28. Frederix GWJ, Severens JL, Hövels AM, Van Hasselt JGC, Hooiveld MJJ, Neven $P$, et al. Real world cost of human epidermal receptor 2-positive metastatic breast cancer patients: A longitudinal incidence-based observational costing study in the Netherlands and Belgium. Eur J Cancer Care (Engl). (10.1111); 2015;24:340-54. [cited 2019 May 6] Available from: http://doi.wiley.com/10.1111/ecc.12266

29. PORDATA. Despesa em cuidados de saúde por habitante (Euro). 2016 [cited 2019 Apr 30]. Available from: https://www.pordata.pt/Europa/Despesa+em+ cuidados+de+saúde+por+habitante+(Euro)-1963

30. Silva LCFF, de Arruda LSM, David WJ, Cruz FJSM, Trufelli DC, del Giglio A. Hormone receptor-negative as a predictive factor for pathologic complete response to neoadjuvant therapy in breast cancer. Einstein (São Paulo). 2019;17. Available from: http://dx.doi.org/

31. NICE. Pertuzumab for the neoadjuvant treatment of HER2-positive breast cancer. Single Technol. Apprais. 2016. Available from: https://www.nice.org. uk/guidance/ta424/resources/pertuzumab-for-the-neoadjuvant-treatment-ofher2positive-breast-cancer-pdf-82604663691973

32. NCPE Ireland. Cost-effectiveness of Pertuzumab (Perjeta) (in combination of trastuzumab and chemotherapy) for the neoadjuvant treatment of adult patients with HER2-positive, locally advanced, inflammatory, or early stage breast cancer at high risk of recurrence. 2016. Available from: http://www. ncpe.ie/wp-content/uploads/2015/09/Summary-Pertuzumab-16.pdf

\section{Publisher's Note}

Springer Nature remains neutral with regard to jurisdictional claims in published maps and institutional affiliations.

\section{Ready to submit your research? Choose BMC and benefit from:}

- fast, convenient online submission

- thorough peer review by experienced researchers in your field

- rapid publication on acceptance

- support for research data, including large and complex data types

- gold Open Access which fosters wider collaboration and increased citations

- maximum visibility for your research: over $100 \mathrm{M}$ website views per year

At BMC, research is always in progress.

Learn more biomedcentral.com/submission 